\title{
¿CÓMO INTERACTÚAN EL AUTOCONCEPTO Y EL RENDIMIENTO ACADÉMICO, EN UN CONTEXTO EDUCATIVO PLURICULTURAL?
}

Francisco Herrera y otros

Facultad de Educación y Humanidades de Ceuta, Universidad de Granada

\section{INTRODUCCIÓN, PROBLEMA Y REVISIÓN TEÓRICA}

Desde las primeras investigaciones sobre el aprendizaje y, por extensión, sobre educación -finales del siglo XIX y primer tercio del XX-, los estudios se centraron exclusivamente en los aspectos cognitivos, desde los más simples a los más complejos, dando como fruto la aparición de numerosas teorías que fueron evolucionando rápidamente, probablemente, debido al gran desconocimiento e incertidumbre propios de cualquier inicio. Con el paso del tiempo -segundo tercio del siglo XX-, paulatinamente, los investigadores descubrieron la importancia de los componentes afectivos y su influencia decisiva en el aprendizaje; tanto que, más de lo conveniente, los estudios se centraron en este nuevo objeto, olvidando casi por completo el ámbito de lo cognitivo, produciéndose entonces un brusco paso de un extremo al opuesto. Hasta que, afortunadamente, los estudiosos tuvieron la feliz idea de conjugar los aspectos cognitivos y los afectivos en común -tercer tercio del siglo XX-, naciendo así el constructo conocido por el nombre de aprendizaje autorregulado (self-regulatedlearning).

Pues bien, en ningún caso, tanto en estudios individualizados sobre los aspectos afectivos, como en conjunción con los cognitivos, se ha abordado la perspectiva de estudio que aquí se presenta, referido a las diferencias en el autoconcepto de los alumnos, en función de la cultura mayoritaria en la que se insertan, en un contexto pluricultural como el de la Ciudad Autónoma de Ceuta.

No obstante, es preciso aclarar que, a lo largo de la historia, la literatura científica ha venido dando diferentes interpretaciones al término autoconcepto, sin haberlo podido delimitar con precisión y claridad (Burns, 1990). El conocimiento de uno mismo es una teoría, es lo que la persona cree de sí mismo y siente sobre sí mismo, aunque lo que crea y sienta no se corresponda con la realidad y, en función de ello, así se comporta. De ahí, que la mayoría de los autores interpreten el autoconcepto globalmente, como conjunto integrado de factores o actitudes relativos al yo, básicamente por tres: cognitivos (pensamientos), afectivos (sentimientos) y conativos (comportamientos); que, de considerarlos individualmente, quizás podrían identificarse de la siguiente manera: el primer factor, como autoconcepto propiamente dicho; el segundo, como autoestima, y, el tercero, como autoeficacia (Ramírez Salguero, 1997).

En cualquier caso, dado que el autoconcepto condiciona el comportamiento, aquí nos interesa conocer particularmente el autoconcepto de los alumnos en el contexto escolar pluricultural de Ceuta, en función de la cultura mayoritaria de los centros educativos a los que pertenecen; de manera que, en base a los hallazgos, se puedan tomar las decisiones más convenientes, en cada caso, de cara a la intervención.

Así pues, se ha planteado el siguiente problema general: ¿Qué efectos provocan los diferentes tipos y niveles de autoconcepto de la población escolar de las Comunidades Ceutíes Cristiana y Musulmana en su rendimiento académico, especialmente en esta última?. 
Este problema general, para su mejor estudio y análisis, hemos decidido descomponerlo en los siguientes problemas específicos:

- ¿Qué tipos y niveles de autoconcepto tiene la población escolar de las comunidades ceutíes cristiana y musulmana, individualizada y diferencialmente, por culturas y sexos?.

- ¿Qué tipos y niveles de rendimiento académico tiene la población escolar de las comunidades ceutíes cristiana y musulmana, individualizada y diferencialmente, por culturas y sexos?.

- ¿Qué relaciones existen entre la autoconcepto y el rendimiento académico de los alumnos de la población escolar de las comunidades ceutíes cristiana y musulmana, individualizada y diferencialmente, por culturas y sexos?

La teorización sobre el autoconcepto han tenido lugar en el ámbito de la fenomenología que Wylie (1961) definió como el estudio de la conciencia directa. Una de las tesis fundamentales de está teoría es que la conducta se ve influenciada no sólo por el pasado y por las experiencias presentes, sino además por los significados personales que cada individuo atribuye a su percepción de esas experiencias. Ese mundo personal privado del individuo es el que más influye sobre su conducta. De este modo, el comportamiento es más que una mera función de lo que nos sucede desde el exterior y es también una consecuencia de cómo creemos que somos (Lewin 1936; Bruner y Goodman, 1947; Raimy (1948); Rogers, 1951; Vinacke, 1952; Kelly, 1955; Judson y Cofer, 1956).

A partir de ahí, aparecen nuevas aportaciones, en principio, en el sentido del autoconcepto como conjunto de actitudes del yo hacia sí mismo (Kretch, Crutchfield y Ballachey, 1962). De igual forma, comienzan a emplearse los términos: autoimagen, autoconfiguración, autovalía o autoeficacia y autoestima, en sentido autoevaluativo o autovalorativo, que cada autor argumenta para distinguirlo y darle mayor peso específico (Rosenberg, 1965; Coopersmith, 1967; Brisset, 1972; Cattell y Child, 1975; etc.).

Burns (1990) interpreta el autoconcepto como conceptualización de la propia persona hecha por el individuo, siendo así considerado como adornado de connotaciones emocionales y evaluativas poderosas, puesto que las creencias subjetivas y el conocimiento fáctico que el individuo se atribuye son enormemente personales, intensos y centrales, en grados variables a su identidad única. Y, respecto a la autoestima o autoevaluación, piensa que es el proceso mediante el cual el individuo examina sus actos, sus capacidades y atributos en comparación a sus criterios y valores personales que ha interiorizado a partir de la sociedad y de los otros significativos, de manera que estas evaluaciones dan una conducta coherente con el autoconocimiento, ubicando el autoconcepto en el ámbito de la actitud.

Esta manera de enfocar la cuestión es muy fructífera, primero, porque permite aplicar métodos aceptados y experimentados para clasificar las actitudes en la medición del autoconcepto y, en segundo lugar, porque centra la atención en el hecho de que el autoconcepto no está compuesto de un elemento único. Los individuos poseen un amplio abanico de autoconceptos en relación a sus percepciones específicas. Así pues, utilizar el término actitudes hacia el yo, en lugar de autoconcepto, acentúa el hecho de la existencia de muchas formas en que la persona puede concebirse a sí misma (Burns, 1990:26).

González y Tourón (1992) lo definen como organización de actitudes que el individuo tiene hacia sí mismo, al modo en que lo hacen Wells y Marwell (1976), Burns (1979), Greenwald y Pratkanis (1984).

Clemes et al. (1994:7) refiriéndose a la autoestima como parte efectiva del autoconcepto, opina que es el punto de partida para el desarrollo positivo de las relaciones humanas, del aprendizaje, de la creatividad y de la responsabilidad personal. Es el <aglutinante> que liga la personalidad del hombre y conforma una estructura 
positiva, homogénea y eficaz. Siempre será la autoestima la que determine hasta qué punto podrá el hombre utilizar sus recursos personales y las posibilidades con las que ha nacido, sea cual fuere la etapa de desarrollo en que se encuentre.

Hablando metafóricamente, podríamos decir que nuestra personalidad es el vehículo que nos conduce por la vida, cuyo motor sería el autoconcepto (aspecto cognitivo), su combustible la autoestima (aspecto afectivo), y su particular forma de conducirse, más o menos acertada, la autoeficacia (aspecto conativo), ya que como pensamos (cognición), sentimos (afectividad) y actuamos (conación).

Un niño con inteligencia superior a la media y con poco autoconcepto puede obtener rendimientos suficientes pero no satisfactorios, mientras que otro de inteligencia media con mucho autoconcepto puede obtener mejores resultados.

Normalmente el niño de poco autoconcepto suele encontrar pocas satisfacciones en el colegio, rápidamente pierde la motivación y el interés y, en cambio, emplea buena parte de sus energías en aquellos aspectos que se relacionan con los sentimientos hacia sí mismo (temores, ansiedades, problemas, relaciones con los demás, etc.).

Con mucha frecuencia, las experiencias capaces de reforzar el autoconcepto están relacionadas con el colegio y, por ello, producen ansiedad con la que el niño lucha continuamente. De esta forma, entra en un círculo vicioso del que cada vez le resulta más difícil salir. El autoconcepto, al margen de su importancia general en el comportamiento escolar, marca todas las manifestaciones de la personalidad, como por ejemplo: el control emocional, la creatividad, las relaciones personales, etc.; siendo patrones de gran influencia el propio autoconcepto de sus referentes próximos: padres y maestros, que se proyectan a quienes están a su alrededor condicionándolos.

Hoy día, la presión social (familia, escuela y ambiente) hace que los alumnos estén envueltos por la obsesión de la competencia académica y por el buen logro académico. Parte del nivel del autoconcepto del escolar viene dado por las aprobaciones o reprobaciones de sus logros escolares dadas por sus referentes significativos, especialmente los padres y profesores. Cuanto mejores calificaciones y más premios obtengan, mayores aprobaciones y, consecuentemente, mayor nivel de autoconcepto tendrán.

Son numerosas las investigaciones que han confirmado la relación positiva significativa entre todas las medidas de autopercepción y logro académico, insistiendo en que la medida del autoconcepto es el mejor pronosticador del logro académico, más aún que las medidas del C.I. y de la aptitud (Jones y Grieneeks, 1970; Purkey, 1970; Machargo, 1989; Díaz Aguado et al., 1992) y que, como comprobaron Musitu et al. (1991), es inferior en los rechazados; de ahí, que también en el factor de "competencia académica" sean significativamente inferiores y, por tanto, tienen también menor capacidad de autocontrol al sentirse torpes y abrumados por las tareas escolares.

Musitu et al. (1983) opinan que, en especial el alumno rechazado, se encuentra ante una serie de exigencias en la escuela que lo desbordan. No puede atenderlas todas por no tener, en gran parte de los casos, capacidad suficiente, por lo que recibe una evaluación negativa del profesor y también de los padres, de lo que se desprende que no está suficientemente motivado como para controlar y asumir todo el conjunto de exigencias de los adultos (padres y profesores).

Los niños en desventaja (especialmente los de las minorías) se consideran como posibles víctimas de 
autoconceptos bajos debido a la discriminación, la pobreza y a las condiciones poco estimulantes del medio. Se supone que estas condiciones conducen a una denigración de la autovalía; pero, a pesar de todo, algunos estudios experimentales revelan que los niños en situación de desventaja no sólo poseen autoconceptos positivos, sino que algunas veces tienen autoconceptos más altos que los grupos aventajados (Soares y Soares, 1969; Trowbridge, 1970; Heiss y Owens, 1972; Rosenberg, 1973, etc.). Estos autores sugieren que esto ocurre así porque los niños pertenecientes a grupos en desventaja están expuestos únicamente a otras personas y vecinos en desventaja, y, de acuerdo con tales expectativas, actúan satisfactoriamente.

Por el contrario, los niños de grupos aventajados tienen expectativas más altas que les sirven de referencia en su motivación. Sólo porque los grupos aventajados miren a los que están en desventaja de una forma negativa, no es suficiente razón para suponer que éstos se vean de la misma forma (Carter, 1979).

En suma, es preciso reiterar que, si bien son muchos los estudios realizados en torno al autoconcepto en el ámbito educativo, no tenemos ninguna constancia de que se haya llevado a cabo alguna investigación en condiciones similares.

\section{HIPÓTESIS}

\section{En consecuencia, se han formulado las siguientes hipótesis:}

\section{General: El autoconcepto de la población escolar de las comunidades ceutíes cristiana y musulmana, afecta significativamente a su rendimiento académico.}

\section{Específicas:}

1. Existen diferencias significativas en los tipos y niveles de autoconcepto de la población escolar de las comunidades ceutíes cristiana y musulmana, individualizada y diferencialmente, por culturas y sexos.

2. Existen diferencias significativas en los tipos y niveles de rendimiento académico de la población escolar de las comunidades ceutíes cristiana y musulmana, individualizada y diferencialmente, por culturas y sexos.

3. Existen relaciones significativas entre la autoconcepto y el rendimiento académico de los alumnos de la población escolar de las comunidades ceutíes cristiana y musulmana, individualizada y diferencialmente, por culturas y sexos.

\section{MÉTODO}

\subsection{Muestra}

La muestra fue extraída de la población escolar de Ceuta de Educación Primaria (segundo y tercer ciclo) y de E.S.O. (Primer ciclo) por muestreo estratificado con afijación proporcional (según el nivel y la etapa educativa, el grupo cultural -cristiano y musulmán-, y la cultura dominante en el centro educativo); garantizando un nivel de confianza del $95,5 \%$ y un margen de error del $3 \%$.

Del total de los sujetos que componen la muestra (1315), el 49,8\% son chicos y el resto chicas, perteneciendo el $50,8 \%$ de ellos a la comunidad cristiana y el resto a la musulmana. 


\subsection{Instrumentos y variables}

El instrumento de evaluación empleado ha sido el Test de Autoconcepto Forma A (AFA), de Musitu, García y Gutiérrez (1991), integrado por 36 frases a las que los sujetos debían responder de acuerdo a su forma de pensar y actuar, en una escala de 1 a 3 , en la que 1 significa -Siempre-, 2 -Algunas veces- y 3 -Nunca-. El valor de su coeficiente de fiabilidad es de 0,86 y permite evaluar las siguientes variables:

- Autoconcepto emocional.

- Autoconcepto familiar.

- Autoconcepto académico.

- Autoconcepto social.

- Autoconcepto global.

\subsection{Resultados}

3.3.1. Diferencias en los tipos y niveles del autoconcepto de la población escolar de las comunidades ceutíes cristiana y musulmana, individualizada y diferencialmente, por culturas y sexos

En este apartado damos cuenta de los resultados obtenidos en los tipos y niveles de autoconcepto de la población escolar de las comunidades ceutíes cristiana y musulmana, individualizada y diferencialmente, por culturas y sexos.

En la tabla 1 se exponen los resultados de la prueba t para el contraste de medias cuando la variable de agrupamiento es el tipo de grupo (cristianos-musulmanes) y la variable agrupada es la puntuación obtenida en los diferentes factores del autoconcepto que se evalúan con el AFA.

\section{Tabla 1}

Prueba t de Student para el contraste de medias de muestras independientes, considerando como variable de agrupamiento el tipo de grupo: cristianos y musulmanes, y como variable agrupada las puntuaciones en los distintos factores del autoconcepto

\begin{tabular}{|c|c|c|c|c|c|c|c|}
\hline \multicolumn{2}{|c|}{ Variables } & \multicolumn{3}{|c|}{ Estadísticos } & \multicolumn{3}{|c|}{ Prueba $t$} \\
\hline Autoconcepto & Grupo & $n$ & _- & ó & $\mathrm{t}$ & g.l. & $p$ \\
\hline Emocional & $\begin{array}{c}\text { Cristianos } \\
\text { Musulmanes } \\
\text { 2Mıııılmanes }\end{array}$ & $\begin{array}{l}668 \\
647\end{array}$ & $\begin{array}{l}53,87 \\
47,14\end{array}$ & $\begin{array}{l}16,22 \\
16,66\end{array}$ & 7,40 & 1313 & 0,000 \\
\hline Familiar & $\begin{array}{l}\text { Cristianos } \\
\text { Musulmanes }\end{array}$ & $\begin{array}{l}668 \\
647\end{array}$ & $\begin{array}{l}56,69 \\
46,26\end{array}$ & $\begin{array}{l}16,93 \\
16,91\end{array}$ & 11,20 & 1313 & 0,000 \\
\hline Académico & $\begin{array}{l}\text { Cristianos } \\
\text { Musulmanes }\end{array}$ & $\begin{array}{l}668 \\
647\end{array}$ & $\begin{array}{l}54,92 \\
49,83\end{array}$ & $\begin{array}{l}17,14 \\
16,46\end{array}$ & 5,50 & 1313 & 0,000 \\
\hline Social & $\begin{array}{l}\text { Cristianos } \\
\text { Musulmanes }\end{array}$ & $\begin{array}{l}668 \\
647\end{array}$ & $\begin{array}{l}66,47 \\
64,50\end{array}$ & $\begin{array}{l}16,61 \\
18,44\end{array}$ & 2,00 & 1313 & 0,042 \\
\hline Global & $\begin{array}{l}\text { Cristianos } \\
\text { Musulmanes }\end{array}$ & $\begin{array}{l}668 \\
647\end{array}$ & $\begin{array}{l}232,0 \\
207,8\end{array}$ & $\begin{array}{l}54,89 \\
59,79\end{array}$ & 7,60 & 1313 & 0,000 \\
\hline
\end{tabular}


En esta tabla se puede observar que todos los valores de $t$ asociados a las diferencias de medias en autoconcepto son estadísticamente significativos $(p<0,05)$.

Así pues, en general, podemos decir que el alumnado cristiano tiene mejor autoconcepto que el musulmán.

En la tabla 2 se exponen los resultados de la prueba $t$ para el contraste de medias cuando la variable de agrupamiento es el sexo (chicos-chicas), del total del alumnado de la muestra, y la variable agrupada es la puntuación obtenida en los diferentes factores del autoconcepto que se evalúan con el AFA.

\section{Tabla 2}

Prueba t de Student para el contraste de medias de muestras independientes, considerando como variable de agrupamiento el sexo: chicos y chicas, del total del alumnado, y como variable agrupada las puntuaciones en los distintos factores del autoconcepto

\begin{tabular}{|c|l|c|c|c|c|c|c|}
\hline \multicolumn{2}{|c|}{ Variables } & \multicolumn{3}{c|}{ Estadísticos } & \multicolumn{3}{c|}{ Prueba $t$} \\
\hline \multirow{2}{*}{ Autoconcepto } & Grupo & $\mathrm{n}$ & - & o & $\mathrm{t}$ & $\mathrm{g} . \mathrm{l}$. & $\mathrm{p}$ \\
\hline \multirow{2}{*}{ Emocional } & Chicos & 655 & 49,82 & 16,43 & $-1,60$ & 1313 & 0,110 \\
& Chicas & 660 & 51,30 & 17,09 & & & \\
\hline \multirow{2}{*}{ Familiar } & Chicos & 655 & 50,90 & 17,57 & $-1,33$ & 1313 & 0,184 \\
& Chicas & 660 & 52,20 & 17,83 & & & \\
\hline \multirow{2}{*}{ Académico } & Chicos & 655 & 50,66 & 17,44 & $-3,35$ & 1313 & 0,000 \\
& Chicas & 660 & 54,16 & 16,39 & & & \\
\hline \multirow{2}{*}{ Social } & Chicos & 655 & 65,70 & 17,71 & 0,41 & 1313 & 0,679 \\
& Chicas & 660 & 65,30 & 17,42 & & & \\
\hline \multirow{2}{*}{ Global } & Chicos & 655 & 217,41 & 58,23 & $-1,65$ & 1313 & 0,098 \\
& Chicas & 660 & 222,75 & 58,88 & & & \\
\hline
\end{tabular}

En esta tabla se puede observar que únicamente los valores de $t$ asociados a las diferencias de medias en autoconcepto académico son estadísticamente significativos $(p<0,05)$, no superando este criterio el resto.

Por lo tanto, en general (para todos los cristianos y musulmanes juntos) y particularmente (en el caso de los cristianos solamente y de los musulmanes solamente), podemos decir que las alumnas tienen mejor autoconcepto académico que los alumnos.

3.3.2. Diferencias en los tipos y niveles de rendimiento académico de la población escolar de las comunidades ceutíes cristiana y musulmana, individualizada y diferencialmente, por culturas y sexos

En este apartado damos cuenta de los resultados obtenidos en los tipos y niveles de rendimiento académico de la población escolar de las comunidades ceutíes cristiana y musulmana, individualizada y diferencialmente, por culturas y sexos.

En la tabla 3 se exponen los resultados de la prueba $t$ para el contraste de medias cuando la variable de agrupamiento es el tipo de grupo (cristianos-musulmanes) y la variable agrupada es la puntuación obtenida en rendimiento académico. 


\section{Tabla 3}

Prueba t de Student para el contraste de medias de muestras independientes, considerando como variable de agrupamiento el tipo de grupo: cristianos y musulmanes, y como variable agrupada las puntuaciones en rendimiento académico

\begin{tabular}{|c|c|c|c|c|c|c|c|}
\hline \multicolumn{2}{|c|}{ Variables } & \multicolumn{3}{|c|}{ Estadísticos } & \multicolumn{3}{|c|}{ Prueba $t$} \\
\hline Rendimiento & Grupos & $\mathrm{n}$ & $\mathbf{x , -}$ & ó & $t$ & g.l. & $p$ \\
\hline Lengua & $\begin{array}{l}\text { Cristianos } \\
\text { Musulmane } \\
\text { s }\end{array}$ & $\begin{array}{l}668 \\
647\end{array}$ & $\begin{array}{l}5,99 \\
4,80\end{array}$ & $\begin{array}{l}1,49 \\
1,47\end{array}$ & 14,6 & $\begin{array}{c}131 \\
3\end{array}$ & 0,000 \\
\hline Matemáticas & $\begin{array}{l}\text { Cristianos } \\
\text { Musulmane } \\
\text { s }\end{array}$ & $\begin{array}{l}668 \\
647\end{array}$ & $\begin{array}{l}5,80 \\
5,33\end{array}$ & $\begin{array}{l}1,62 \\
1,38\end{array}$ & 5,70 & $\begin{array}{c}131 \\
3\end{array}$ & 0,000 \\
\hline CC. Naturales & $\begin{array}{c}\text { Cristianos } \\
\text { Musulmane } \\
\text { s }\end{array}$ & $\begin{array}{l}668 \\
647\end{array}$ & $\begin{array}{l}6,03 \\
5,47\end{array}$ & $\begin{array}{l}1,36 \\
1,30\end{array}$ & 7,60 & $\begin{array}{c}131 \\
3\end{array}$ & 0,000 \\
\hline CC. Sociales & $\begin{array}{l}\text { Cristianos } \\
\text { Musulmane } \\
\text { s }\end{array}$ & $\begin{array}{l}668 \\
647\end{array}$ & $\begin{array}{l}5,81 \\
5,06\end{array}$ & $\begin{array}{l}1,51 \\
1,56\end{array}$ & 8,80 & $\begin{array}{c}131 \\
3\end{array}$ & 0,000 \\
\hline Global & $\begin{array}{l}\text { Cristianos } \\
\text { Musulmane } \\
\text { s }\end{array}$ & $\begin{array}{l}668 \\
647\end{array}$ & $\begin{array}{l}5,69 \\
4,82\end{array}$ & $\begin{array}{l}1,70 \\
1,49\end{array}$ & 9,90 & $\begin{array}{c}131 \\
3\end{array}$ & 0,000 \\
\hline
\end{tabular}

En esta tabla se puede observar que todos los valores de $t$ asociados a las diferencias de medias en rendimiento académico son estadísticamente significativos $(p<0,05)$.

Así pues, en general, podemos decir que el alumnado cristiano tiene mejor rendimiento académico que el musulmán, tanto por áreas curriculares como globalmente.

Asimismo, destacar que, en general (para todo el alumnado, cristiano y musulmán juntos), en función del sexo, no se aprecian diferencias estadísticamente significativas.

Por otra parte, manifestar que en cuanto a las diferencias por sexo, dentro del alumnado cristiano, no se han hallado diferencias estadísticamente significativas entre los chicos y las chicas en su rendimiento académico; mientras que, dentro del alumnado musulmán, tan sólo se han encontrado diferencias estadísticamente significativas en el caso del rendimiento en el Área de Ciencias Sociales, a favor de las chicas (p 0,000).

3.3.3. Relaciones existentes entre el autoconcepto y el rendimiento académico de los alumnos de la población escolar de las comunidades ceutíes cristiana y musulmana, individualizada y diferencialmente, por culturas y sexos

Según los datos encontrados se observa que, solamente en el caso de la comunidad escolar ceutí musulmana, el rendimiento académico global del alumnado está determinado por su autoconcepto académico, que explica el $12,34 \%$ de la varianza de éste ( $\hat{a}=0,1234, t=2,930$ y $p=0,0035)$; pero, en el caso de los alumnos cristianos solamente o en el caso del conjunto de alumnos cristianos y musulmanes, no destaca ningún predictor del rendimiento académico.

\section{CONCLUSIONES Y DISCUSIÓN}


Tras el análisis de los datos obtenidos podemos concluir lo siguiente:

\subsection{Respecto a la hipótesis general}

Podemos afirmarla en el sentido que indican las hipótesis específicas (subhipótesis) planteadas: El autoconcepto de la población escolar de las comunidades ceutíes cristiana y musulmana, afecta significativamente a su rendimiento académico.

\subsection{Respecto a la primera hipótesis específica}

Podemos afirmarla en el siguiente sentido:

1. En el caso de la población escolar de la comunidad ceutí, por culturas: cristiana y musulmana.

Existen diferencias estadísticamente significativas en todos los factores del autoconcepto analizados a favor de los cristianos; es decir, éstos son los que parecen tener mejor autoconcepto.

2. En el caso de la población escolar de la comunidad ceutí, por sexos: chicos y chicas.

En general, para todos los alumnos cristianos y musulmanes juntos o individualmente por culturas, podemos decir que sólo existen diferencias estadísticamente significativas en el autoconcepto académico a favor de las chicas.

4.3. Respecto a la segunda hipótesis específica

Podemos afirmarla en el siguiente sentido:

1. En el caso de la población escolar de la comunidad ceutí, por culturas: cristiana y musulmana.

Existen diferencias estadísticamente significativas en el rendimiento académico en todas las áreas curriculares y globalmente a favor de los alumnos cristianos.

2. En el caso de la población escolar de la comunidad ceutí, por sexos: chicos y chicas.

En general, para todo el alumnado cristiano y musulmán junto, en cuanto a las diferencias en el rendimiento académico por sexo, podemos decir que no existen diferencias estadísticamente significativas entre chicos y chicas.

En el alumnado cristiano, no se han hallado diferencias estadísticamente significativas entre los chicos y las chicas en su rendimiento académico.

En el alumnado musulmán, tan sólo se han encontrado diferencias estadísticamente significativas en el caso del rendimiento en el Área de Ciencias Sociales, a favor de las chicas.

\subsection{Respecto a la tercera hipótesis específica}

Podemos afirmarla en el siguiente sentido:

Tan sólo en el caso de los alumnos musulmanes aparece como predictor del rendimiento a académico el autoncepto académico.

Finalmente, aunque nuestra investigación tiene un contexto pluricultural no equiparable al de otras investigaciones realizadas, hacer constar que bien podría confirmar, de alguna forma, la opinión de Musitu et al. (1991) en el sentido de que el autoconcepto de los rechazados es inferior; de ahí, que también lo sea su factor 
de competencia académica, y, por tanto, también su capacidad de autocontrol, al sentirse torpes y abrumados por las tareas escolares. Interpretando aquí como rechazados a los niños en desventaja (especialmente los de las minorías), posibles víctimas de autoconceptos bajos debidos a la discriminación, la pobreza, a las condiciones poco estimulantes de su entorno y a su comparación con los que gozan de mejor situación.

\section{BIBLIOGRAFÍA}

BAndurA, A., Self-efficacy. Toward a unifying theory of behavioral change. Psychological Review, 84, 1977, 191-215.

BuRNs, R.B., El autoconcepto. Bilbao, Ega, 1990.

Clemes, H.; BeAn, R. y ClaRK, A., Cómo desarrollar la autoestima en niños y adolescentes. Madrid, Debate, 1994.

COOPERSMITH, S., The adolescents of self-esteem. San Francisco, Freeman, 1977.

Díaz Aguado, M.J.; Martínez Arias, R. y Baraja, A., Educación y desarrollo de la tolerancia. Programas para favorecer la integración educativa en contextos étnicamente heterogéneos. I, II y III - Teoría. Madrid, M.E.C., 1992.

GonzÁLEZ, M.C. y Tourón, J., Autoconcepto y rendimiento escolar. Pamplona, EUNSA, 1992.

MACHARGO, J., El profesor y el autoconcepto de sus alumnos. Madrid, Escuela Española, 1989.

Musitu, G.; García, F. y Gutiérrez, M., Autoconcepto, Forma - A (AFA). Madrid, TEA, 1991.

Musitu, G.; RomÁn, J.M. y MARTORELL, M.C., Autoconcepto e integración social en el aula. Tarragona, Universitas Tarraconensis, 1983.

Ramírez SALGUeRo, M.I., La adaptación como factor de rendimiento de la población escolar de la comunidad musulmana ceutí. Ceuta, UNED, 1997. 